Original paper

\title{
Monte Carlo simulation of COMS ophthalmic applicators loaded with Bebig I25.S16 seeds and comparison with planning system predictions
}

\author{
Héctor Miras ${ }^{\mathrm{a}, *}$, José A. Terrón ${ }^{\mathrm{a}}$, Antonio M. Lallena ${ }^{\mathrm{b}}$ \\ ${ }^{a}$ Servicio de Radiofisica Hospitalaria, Hospital Universitario “Virgen Macarena”, Avda. Doctor Fedriani, 3, E-41009 Sevilla, Spain \\ ${ }^{\mathrm{b}}$ Departamento de Física Atómica, Molecular y Nuclear, Universidad de Granada, E-18071 Granada, Spain
}

\section{A R T I C L E I N F O}

\section{Article history:}

Received 14 April 2012

Received in revised form

29 June 2012

Accepted 9 July 2012

Available online 31 July 2012

\section{Keywords:}

Ophthalmic applicators

Monte Carlo simulation

I25.S16 Bebig seed

\begin{abstract}
A B S T R A C T
Purpose: To simulate the Bebig model I125.S16 source and obtain AAPM Task Group Report 43 brachytherapy dosimetry parameters for comparison to consensus and previously published values. The seed model will then be incorporated into a Monte Carlo model of COMS eye plaques and simulation results will be used for seed-carrier set modeling in a commercial planning system.

Methods: PENELOPE was used to simulate the seed and the applicators for different sizes and loading levels. The corresponding TG-43U1 dosimetric parameters of the seed were calculated. Bebig Plaque Simulator was used.

Results: The air kerma strength, the dose rate constant and the radial dose and 2D anisotropy functions found showed a good agreement with those published by other authors. Dose distributions were determined for the 12 and $20 \mathrm{~mm}$ COMS plaques loaded with a single seed and for the $12 \mathrm{~mm}$ plaque fully loaded. The plaque effect on the eye dose and the interseed absorption were evaluated. If the plaque is loaded with a single seed, the dose in the central axis reduces about $10 \%$ at $5-6 \mathrm{~mm}$ depth with respect to the case in which the plaque is not present. This reduction does not depend on the plaque size. When the plaque is fully loaded, an additional reduction in the dose with respect to the dose in water is observed mainly due to the effect of the Silastic carrier. The mean dose reduction in the central axis of the $12 \mathrm{~mm}$ plaque due to the interseed absorption was $0.5 \%$. A new physics file for the planning system was created with the results obtained from the simulations. Results obtained using this adapted model for the $12 \mathrm{~mm}$ plaque fully loaded agreed with the corresponding simulation. Dose rate at the prescription point differs $4.7 \%$ when the adapted model is used instead of the default model.

Conclusions: Simulation results for COMS plaques are consistent with those published for other seeds. The planning system studied appears as a good tool for dose calculation in ophthalmic brachytherapy treatments. The new physics model, built up from Monte Carlo results, has been commissioned by comparing calculations made with the planning system to those obtained from Monte Carlo simulations. (c) 2012 Associazione Italiana di Fisica Medica. Published by Elsevier Ltd. All rights reserved.
\end{abstract}

\section{Introduction}

Collaborative Melanoma Ocular Study (COMS) is a multicenter cooperative group created during 1980's to evaluate the role of radiotherapy treatment in the management of choroidal melanoma. The COMS randomized trial compared enucleation against episcleral brachytherapy for medium-sized tumors and showed that survival rates for both techniques were equivalent [1].

The COMS protocol specified using I-125 sources in a set of standardized plaque applicators. Nowadays other gamma radionuclide, such as ${ }^{103} \mathrm{Pd}$ and ${ }^{131} \mathrm{Cs}$, and the beta emitter ${ }^{106} \mathrm{Ru} /{ }^{106} \mathrm{Rh}$, are also utilized [2].

\footnotetext{
* Corresponding author. Tel.: +3495 5008940.

E-mail address: hector.miras@gmail.com (H. Miras).
}

Initially, COMS recommended to calculate dose distributions considering seeds as point sources in a homogeneous water medium. However, Chiu-Tsao et al. [3] and de la Zerda et al. [4] reported that this approximation may lead to deviations of around $10 \%$ in the dose at the regions of interest due to the presence of gold and Silastic materials in the COMS eye plaque.

In 2003, a reanalysis of COMS trial data was published [5]. In this work it was concluded that the inclusion of various factors needed to take into account the anisotropy, the lineal source approximation, the effect of the gold plaque and the attenuation of the Silastic seed carriers resulted in a significant reduction of the dose at the structures of interest. In that work, dose calculations were carried out using the Bebig (Eckert \& Ziegler Isotope Products $\mathrm{GmbH}$, Berlin, Germany) Plaque Simulator system (BPSS) using a constant dose correction factor $T=0.9$. Later, a new version (v. 5) of BPSS was 
introduced to incorporate additional scatter and attenuation correction factors, $T(r)$, depending on the distance $r$ between the calculation point and the seed, and account for the path length of primary radiation in the Silastic seed carrier [6], together with the inclusion of dosimetric data for ${ }^{125} \mathrm{I}$ and ${ }^{103} \mathrm{Pd}$ seed models taken from the TG-43U1 report [7].

Ophthalmic brachytherapy with Bebig I25.S16 Isoseed model, together with the use of COMS applicators, was implemented in our center last year. Since then, a number of Monte Carlo (MC) simulations have been performed in order to determine the dose distributions for the I25.S16 seeds in COMS plaques using the seed coordinates specified in the BPSS and to verify its calculation algorithm. The results of the simulation were compared with the calculated doses using BPSS (v. 5.3.8).

The information available from Bebig indicates that the I25.S16 seed model is geometrically the same than the earlier I25.S06 model but it is available with higher source strengths suitable for eye plaque treatment. In fact, the I25.S16 dosimetric data included in the default physics file used by BPSS are exactly the same as those of the I25.S06 model. The dosimetric parameters for the I25.S06 seed have been reported using different MC codes (MCPT, PTRAN and EGSnrc) [8-10]. In this work, we used PENELOPE code [11] to simulate a single seed in a homogeneous water phantom and in COMS eye plaques and some configurations of these plaques including several seeds. The simulation results for a single seed in homogeneous water were compared with the TG-43U1 dosimetric parameters compiled in reference [7] and those published by other authors [8-10].

In the present work, the I25.S16 seed model has been simulated in COMS plaques for the first time. Thomson and Rogers [12] performed simulations with other different seed models in COMS plaques to study the effects due to the plaque-carrier set. Here, the procedures used by these authors were followed to study dose distribution variations with respect to those obtained when the seed is situated inside a homogeneous water phantom.

Finally, from the simulation results, a model has been created for the plaque-seed set in BPSS. Doses calculated from MC simulation were compared to those predicted by BPSS in order to verify the calculation algorithm. Differences obtained when using the new model and the default one for the I25.S16 seed have also been evaluated.

\section{Materials and methods}

PENELOPE code (2008 version)

MC simulations have been performed using the 2008 version of PENELOPE code (PENetration and Energy Loss of Positrons and Electrons) [11]. Only photon transport has been considered in this study. Photoelectric cross sections were obtained from EPDL97 [13]. Born approximation was used to estimate Rayleigh scattering [14], together with non relativistic form factors from Hubbell et al. [15], whilst Compton scattering was described using cross sections obtained from the relativistic impulse approximation [16].

Track-length estimators, considered by other authors in previous works as a variance reduction technique, were not used in the simulations that we have carried out.

\section{Geometry and materials}

As indicated above, the models I25.S16 and I25.S06 have the same design according to the information provided by the manufacturer. Material and geometry of the I25.S06 seed were described by Hedtjärn et al. [8] and by Taylor and Rogers [17]. A scheme of the geometry used in our simulations, including sources and a COMS applicator is shown in Fig. 1.

The source consists of ${ }^{125}$ I (half-life of 59.4 days) uniformly distributed in an alumina porous ceramic $\left(\mathrm{Al}_{2} \mathrm{O}_{3}\right)$ with $2.88 \mathrm{~g} \mathrm{~cm}^{-3}$ density. The active material is in a cylindrical tube with $0.22 \mathrm{~mm}$ and $0.6 \mathrm{~mm}$ of internal and external diameters, respectively, and $3.5 \mathrm{~mm}$ of length. A gold cylindrical marker (with $19.32 \mathrm{~g} \mathrm{~cm}^{-3}$ density) is situated in the inner space with $0.17 \mathrm{~mm}$ diameter and $3.5 \mathrm{~mm}$ length.

Encapsulation is composed of a titanium tube with $4.54 \mathrm{~g} \mathrm{~cm}^{-3}$ density, $0.05 \mathrm{~mm}$ thickness and $0.8 \mathrm{~mm}$ external diameter with rounding edges. These have been simulated as outer hemispheres with $0.4 \mathrm{~mm}$ of radius, centered at $1.88 \mathrm{~mm}$ from the seed center and an internal hemisphere with $0.889 \mathrm{~mm}$ radius, centered at $0.951 \mathrm{~mm}$ from the center of the seed. The total length of the seed is $4.56 \mathrm{~mm}$.

COMS ophthalmic applicators consist of a plaque of a gold alloy named Modulay (77\% Au, $14 \% \mathrm{Ag}, 8 \% \mathrm{Cu}$ and $1 \% \mathrm{Pd}$, with $15.8 \mathrm{~g} \mathrm{~cm}^{-3}$ density) and a seed carrier of a silicon material known as Silastic (0.63\% H, 24.9\% C, 28.9\% O, 39.9\% Si and 0.005\% Pt, with $1.12 \mathrm{~g} \mathrm{~cm}^{-3}$ density), which includes different holes to allocate the seeds. The Silastic carrier is coupled to the inner part of the plaque. The internal curvature radius is $12.3 \mathrm{~mm}$ and adapts to a spherical eye model of $24.6 \mathrm{~mm}$ diameter.

The plaque is formed of a piece of spherical skullcap of $0.5 \mathrm{~mm}$ thickness and $15.05 \mathrm{~mm}$ external curvature radius. It ends in a cylindrical collimator with a thickness of $0.5 \mathrm{~mm}$ and a variable height within 2.4 and $4 \mathrm{~mm}$, according to the plaque size. The diameter of the internal cylindrical collimator determines the plaque size, which can be chosen among 10, 12, 14, 16, 18, 20 and $22 \mathrm{~mm}[3,18]$. In what follow we will use this size to refer to the plaques considered in the simulations. Specifically, results for the 12 and $20 \mathrm{~mm}$ plaques are presented.

Seeds are distributed in the seed carrier in rings centered with respect to the central axis of the plaque and over a spherical surface with radius $13.7 \mathrm{~mm}$. In this way the centers of these rings coincide with those of the plaque and the inner surface of the seed carrier. With this set up, the seed is $1.4 \mathrm{~mm}$ apart from the inner surface of the seed carrier (sclera external surface).

\section{Simulation of the Bebig I25.S06/I25.S16 seeds}

The ${ }^{125}$ I was assumed to emit photons of energies (and relative intensities) 27.20 (0.406), 27.47 (0.757), 30.98 (0.202), 31.71 (0.04) and $35.49 \mathrm{keV}(0.067)$, with an average energy of $28.37 \mathrm{keV}[7,19]$. Only photon transport was considered in all simulations. The energy of the emitted photons allows considering the kerma-to-dose approximation since the range of secondary electrons is shorter than the voxel sizes used.

The seed was simulated in water and in air to obtain the TG-43U1 parameters. Seed geometry presents axial symmetry and symmetry with respect to the transverse plane that includes the

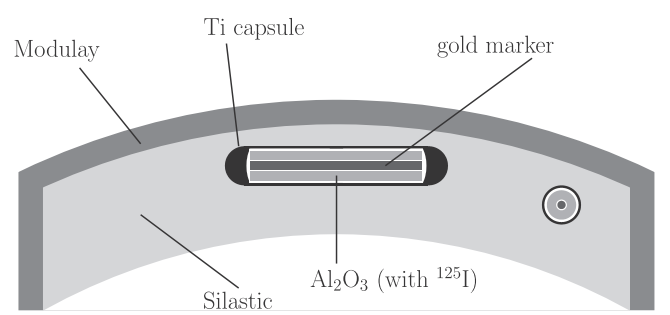

Figure 1. Transverse section of the COMS 12 plaque loaded with Bebig IsoSeed I25.S16 seeds. 
center of the seed (the $z=0$ plane). Scoring voxels chosen for the simulations were defined in cylindrical $\left(\rho=\sqrt{x^{2}+y^{2}, z}\right)$ or spherical $\left(r=\sqrt{x^{2}+y^{2}+z^{2}, \theta}\right)$ coordinates. In both cases symmetry over the azimuthal angle was assumed. The 2D anisotropy function was calculated averaging each voxel with its symmetric with respect to the $z=0$ plane.

To calculate the air kerma strength per activity unit, $s_{K}=S_{K} / A$, two simulations of the seed into a $3 \times 3 \times 3 \mathrm{~cm}^{3}$ dry air cube were carried out. In the first one, a total of $10^{10}$ histories were generated and voxels defined in spherical coordinates with $\Delta r=2 \mathrm{~cm}$ and $\Delta \theta=10^{\circ}$ were used. In the second one, voxels with $\Delta r=1 \mathrm{~cm}$ and $\Delta \theta=3^{\circ}$ were considered in order to determine if $s_{K}$ depends on the voxel size. To compensate the increase in the statistical uncertainty due to the smaller voxel size, air density was increased by a factor 10 . This does not introduce any bias because $s_{K}$ is defined in vacuum and then, it is necessary to correct the kerma distribution for the attenuation in the media. This correction is made by fitting the function [8]

$\dot{K}(d) d^{2}=S_{K}(1+\alpha d) \exp (-\mu d)$

to the simulation results. Here $\dot{K}$ is the kerma rate, $d$ the distance to the source, $S_{k}$ the air kerma strength, $\alpha$ is a constant that takes into account the scatter radiation and $\mu$ is related to primary radiation attenuation. The same procedure described above was performed for a ${ }^{125}$ I point source in order to determine the relationship between the apparent and the actual activities.

Finally, the seed was simulated in vacuum in the center of a cylindrical detection ring $(100 \mathrm{~cm}$ radius and $10 \mathrm{~cm}$ height) centered in the transversal plane with its axis coincident with that of the seed. The energy spectral fluence, $\Phi_{E}$, at the inner surface of this ring was obtained. The kerma rate can be calculated as

$\dot{K}=\int \mathrm{d} E E \Phi_{E}(E) \tilde{\mu}_{\mathrm{tr}}(E)$,

with $\tilde{\mu}_{\mathrm{tr}}(E)$ the mass energy transfer coefficient in air. In these conditions ( $d=100 \mathrm{~cm}$ and vacuum) this parameter coincides with $S_{K}$.

In all the simulations in air, the absorption energy for photons was fixed to $5 \mathrm{keV}$. In this way, the contribution coming from titanium characteristic X-rays was eliminated, as recommended in TG-43U1 [7]. When the medium was water, photons with energies down to $1 \mathrm{keV}$ were considered.

Calculations of the dose rate constant and the radial dose and 2D anisotropy functions were based on simulations performed situating the source in a water sphere of $50 \mathrm{~cm}$ diameter. For the radial dose function, voxels defined in cylindrical coordinates with $\Delta z=1 \mathrm{~mm}$ and $\Delta \rho=0.5 \mathrm{~mm}$, in the range $1 \mathrm{~cm}<\rho \leq 10 \mathrm{~cm}$, and with $\Delta z=0.2 \mathrm{~mm}$ and $\Delta \rho=0.1 \mathrm{~mm}$, in the range $\rho \leq 1 \mathrm{~cm}$, were considered. For the 2D anisotropy function, voxels in spherical coordinates with $\Delta \theta=1^{\circ}$ and $\Delta r=0.5 \mathrm{~mm}$, for $r \leq 1 \mathrm{~cm}$, $\Delta r=1.5 \mathrm{~mm}$, for $1<r \leq 5 \mathrm{~cm}$, and $\Delta r=2.5 \mathrm{~mm}$, for $5<r \leq 10 \mathrm{~cm}$, were used.

\section{Simulation of the COMS plaques}

The effects of the presence of the plaque and the seed carrier on the dose distribution were also studied and the differences with the approximation of homogeneous water medium were evaluated. To account for these effects, the 12 and $20 \mathrm{~mm}$ plaques were simulated in a $20 \times 20 \times 20 \mathrm{~cm}^{3}$ water phantom.

Figure 2 shows the coordinate system for the $12 \mathrm{~mm}$ plaque. The $Z$ axis corresponds to the plaque central axis and is positive towards the inner part of the eyeball. The coordinate reference system has

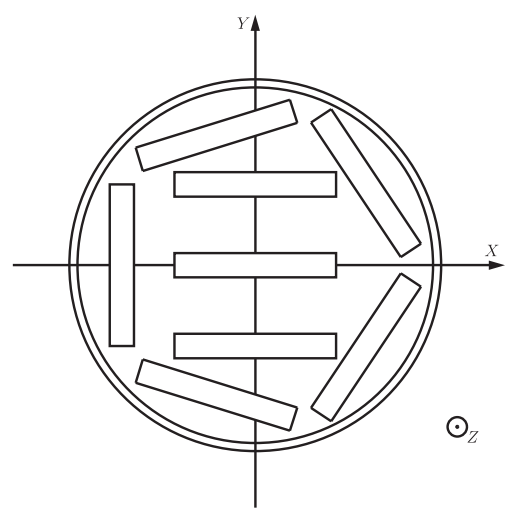

Figure 2. Coordinate system defined for the $12 \mathrm{~mm}$ COMS plaque.

been defined with the origin at the plaque central axis ( $Z$ axis), $1 \mathrm{~mm}$ above the internal surface of the seed carrier. It is placed in the inner surface of the sclera and in the tumor base.

Interseed absorption in the plaque was also evaluated by simulating the $12 \mathrm{~mm}$ plaque fully loaded with eight seeds. The resulting dose distribution was compared to that obtained by adding the different distributions due to a single seed in each possible position in the plaque, with the rest of the seed slots unoccupied. In this case, the voxels were defined in Cartesian coordinates, with dimensions $1 \times 1 \times 1 \mathrm{~mm}^{3}$. The number of histories in each simulation was $10^{10}$, enough to reach a relative uncertainty of $0.5 \%$ (with a coverage factor $k=2$ ) at the prescription point $(z=5 \mathrm{~mm})$.

\section{Plaque and seed carrier model for BPSS}

The dose calculation algorithm implemented in BPSS [6] takes into account the radial dose and the 2D anisotropy functions corresponding to each particular seed. These functions are tabulated with data extracted from TG-43U1 [7]. The attenuation in the Silastic carrier is also considered and the lack of backscatter due to absorption in the plaque is included by means of two correction factors, $t(d, \mu)$ and $T(r)$, that are functions of the primary radiation track-length in the seed carrier, $d$, and the distance between the seed center and the calculation point, $r$. The factor $t(d, \mu)$ is analytical, while $T(r)$ is tabulated with data taken from TLD measurements performed by Chiu-Tsao et al. [3] for the seed model 6711. All these parameters are kept in the physics file of BPSS and can be edited.

A new physics file was created for the model COMS plaque and the I25.S16 seed, using the results for air kerma strength, dose rate constant, radial dose function, 2D anisotropy function and $T(r)$ factor obtained from the MC simulations performed here. The new $T(r)$ function introduced in the BPSS is the ratio between plaque and water dose distributions in the central axis of a $12 \mathrm{~mm}$ plaque with a single seed in the central position, considering the difference in the origin of $z$ coordinate. A single $T(r)$ function could be used for all plaques since, as it will be shown in the results section, it has no significant dependence on the plaque size.

The new model was validated by calculating the dose distribution corresponding to the $12 \mathrm{~mm}$ plaque fully loaded and comparing the BPSS results with those of the MC simulation for the same configuration.

\section{Results and discussion}

Throughout the text, statistical uncertainties are given with a coverage factor $k=2$. In text and tables, they have been expressed 
with a number between parentheses that affects the last figure, i.e., 0.0218 (2) means $0.0218 \pm 0.0002$. In some of the figures, these uncertainties are smaller than the symbols used to represent the corresponding simulation data.

\section{I25.S06/I25.S16 seed simulation}

The results obtained for $s_{K}$ with the three different procedures described above are compared in Table 1 . The value obtained in "normal air" is within the uncertainty of those found in "dense air" and in vacuum. A $2 \%$ difference was found between our results and those quoted by Hedtjärn et al. [8] and by Sahoo and Palani Selvam [9].

If a point source is considered, the value of $s_{K}$ coincides with the air-kerma rate constant $\Gamma_{\delta}$, where $\delta$ labels the photon absorption energy. In our case, the value calculated was

$$
\begin{aligned}
\Gamma_{5 \mathrm{keV}} & =1.3201 \mathrm{cG} y \mathrm{~cm}^{2} \mathrm{~h}^{-1} \mathrm{mCi}^{-1} \\
& =0.03568 \mu G y \mathrm{~m}^{2} \mathrm{~h}^{-1} \mathrm{MBq}^{-1}
\end{aligned}
$$

following the same procedure than for $s_{K}$ (simulation of a point source in air, including attenuation and scatter corrections). The relation among actual and apparent activities, which was calculated as the ratio $\Gamma_{5 \mathrm{kev}} / S_{K}$, gave 1.3810 (8).

The dose rate constant obtained in our simulations is compared to that found by other authors in Table 2 . The value found in this work was in good agreement with that published by Sahoo and Palani Selvam [9], using the EGSnrc code, but differs from those calculated by Taylor and Rogers [20], also with EGSnrc, and by Hedtjärn et al. [8], with PTRAN. The experimental result found by Patel and Chiu-Tsao [21] is consistent, within the uncertainty level, with all the results shown in the table.

Radial dose function results are shown in Table 3 and Fig. 3. Following the procedure proposed by Taylor and Rogers [10,20], we first fitted a function

$g_{\mathrm{fit}}(r)=\left(\sum_{k=0}^{5} a_{k} r^{k-2}\right) \exp \left(-a_{6} r\right)$

to the data obtained from the MC simulations and then the values of $g_{\mathrm{L}}(r)$ were normalized in such a way that $g_{\text {fit }}(r=1 \mathrm{~cm})=1$. This reduces the influence of the uncertainty in the dose rate around $r=1 \mathrm{~cm}$ on the radial function values in the other positions.

The 2D anisotropy function obtained in our simulation is given in Table 4. Figure 4 shows this function evaluated at $r=0.25$ and $0.5 \mathrm{~cm}$. Our results (dots) are compared to those of Hedtjärn et al. [8] (solid curves) and Taylor and Rogers [20] (dashed curved). These radial distances have been selected to show results in the range of interest to ophthalmic brachytherapy treatments.

The radial dose function shows a very good agreement with data from Taylor and Rogers [20] even for the smallest values of $r$ (below $0.1 \mathrm{~cm}$ ). For $r<7 \mathrm{~cm}$, the differences between the fitting function reported by these authors and that obtained in the present work

\section{Table 1}

Comparison of $s_{K}$ values obtained in this work for the I25.S06/I25.S16 seed with the values published by different authors.

\begin{tabular}{lll}
\hline MC code & $s_{K}\left[\mathrm{U} \mathrm{mCi}^{-1}\right]$ & \\
\hline PTRAN & 0.935 & Hedtjärn et al. [8] \\
EGSnrc & $0.934(1)$ & Sahoo and Palani Selvam [9] \\
PENELOPE & $0.955(2)$ & This work (normal air) \\
PENELOPE & $0.9559(5)$ & This work (dense air) \\
PENELOPE & $0.9539(3)$ & This work (vacuum) \\
\hline
\end{tabular}

Table 2

Comparison of constant dose rate values calculated in this work for the I25.S06/ I25.S16 seed with values published by different authors.

\begin{tabular}{lll}
\hline MC code & $\Lambda\left[\mathrm{cGy} \mathrm{h}^{-1} \mathrm{U}^{-1}\right]$ & \\
\hline PTRAN & $1.002-1.010$ & Hedtjärn et al. [8] \\
EGSnrc & $0.994(2)$ & Sahoo and Palani Selvam [9] \\
EGSnrc & $1.011(2)$ & Taylor and Rogers [17] \\
EGSnrc & $1.016(3)$ & Taylor and Rogers [17] \\
PENELOPE & $0.995(3)$ & This work \\
TLD (exp) & $1.033(66)$ & Patel and Chiu-Tsao [21] \\
Consensus & 1.012 & Rivard et al. [7] \\
\hline
\end{tabular}

are below $0.2 \%$. For larger $r$ values, these differences grow up reaching $1.25 \%$ for $r=10 \mathrm{~cm}$. The agreement with the results of other works $[8,9]$ is also good, the differences being smaller than $1 \%$.

The 2D anisotropy function obtained here agrees, within the statistical uncertainties, with that quoted by Hedtjärn et al. [8]. However, there are significant differences with the results of Taylor and Rogers [20] mainly for small angles $\left(\theta<10^{\circ}\right)$ and distances $(0.25 \mathrm{~cm} \leq r \leq 0.5 \mathrm{~cm})$. For larger angles and distances the differences are below $2 \%$. These differences could be ascribed to differences in the size and shape of the accumulation voxels and also to the consideration of track-length estimators, that have not been used in this work.

\section{COMS plaque simulation}

Changes in the dose distribution due to the gold plaque and the Silastic carrier are illustrated in Fig. 5 where the ratio between the dose in the central axis obtained with and without plaque is plotted for various configurations. As we can see, when the plaque includes a single seed, these changes are non-negligible and the dose reduces about $10 \%$ at a depth of $5-6 \mathrm{~mm}$. This reduction is similar for the 12 (open circles) and 20 (solid circles) mm plaques. COMS plaques compounds have atomic number higher than water and then most of radiation particles that reach the plaque are absorbed by photoelectric effect. Plaques act not only as seed carriers but also as a shielding to protect the posterior and lateral regions of the eye. The presence of the set plaque-Silastic carrier has also a non-negligible influence on the dose distribution in the eye. This is due to the attenuation of the radiation in the Silastic carrier and to a reduction in the scatter radiation linked to the absorption of most of the primary radiation incident on the Modulay plaque.

Table 3

I25.S06/I25.S16 Bebig seed radial function obtained in the MC simulations with PENELOPE.

\begin{tabular}{llcl}
\hline $\mathrm{r}[\mathrm{cm}]$ & $g_{L}(r)$ & $r[\mathrm{~cm}]$ & $g_{L}(r)$ \\
\hline 0.05 & $1.098(2)$ & 1.50 & $0.935(1)$ \\
0.06 & $1.045(2)$ & 2.00 & $0.858(1)$ \\
0.07 & $1.024(2)$ & 2.50 & $0.774(2)$ \\
0.08 & $1.015(2)$ & 3.00 & $0.692(2)$ \\
0.09 & $1.012(2)$ & 3.50 & $0.615(2)$ \\
0.10 & $1.013(2)$ & 4.00 & $0.543(2)$ \\
0.15 & $1.023(2)$ & 4.50 & $0.475(2)$ \\
0.20 & $1.031(2)$ & 5.00 & $0.415(2)$ \\
0.25 & $1.038(2)$ & 5.50 & $0.363(2)$ \\
0.30 & $1.037(3)$ & 6.00 & $0.316(1)$ \\
0.40 & $1.041(3)$ & 6.50 & $0.273(1)$ \\
0.50 & $1.036(4)$ & 7.00 & $0.236(1)$ \\
0.60 & $1.036(4)$ & 7.50 & $0.205(1)$ \\
0.70 & $1.030(4)$ & 8.00 & $0.175(1)$ \\
0.75 & $1.023(4)$ & 8.50 & $0.151(1)$ \\
0.80 & $1.020(4)$ & 9.00 & $0.130(1)$ \\
0.90 & $1.012(5)$ & 9.50 & $0.112(1)$ \\
1.00 & $1.001(1)$ & 10.00 & $0.096(1)$ \\
\hline
\end{tabular}




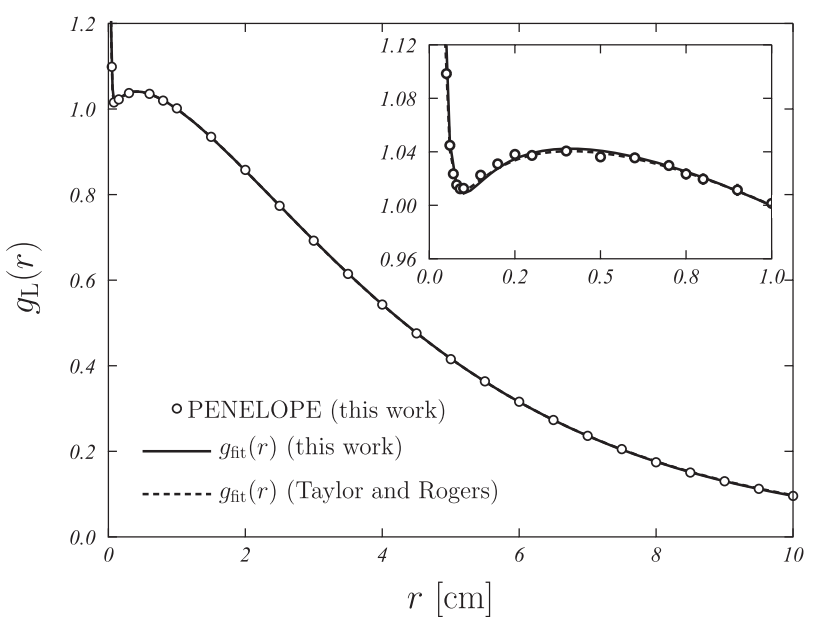

Figure 3. Radial function found in the simulation for the I25.S06/I25.S16 Bebig seed (dots) compared to the fitting function in Equation (4) obtained from our Monte Carlo results (solid curve) and with the fit quoted by Taylor and Rogers (dashed curve) [20].

The dose reduction in central axis (see Fig. 5) for the plaque loaded with a single seed does not depend significantly on the plaque size. However, an appreciable difference with respect to the single seed configuration can be observed when the $12 \mathrm{~mm}$ plaque is fully loaded (open squares). This is due to the fact that in this situation, the radiation emitted in any direction other than that defined by the transverse seed axis, follows a trajectory with a longer path length across the Silastic carrier and with a bigger attenuation.
In principle, interseed absorption should not be very relevant since the seed configuration in the plaque is defined to prevent them from shadowing. Simulation results confirm this assumption for ophthalmic brachytherapy treatments. The average dose reduction in the central axis is $0.5(3) \%$, a value consistent with that quoted by Thomson et al. [18]. It is worth mentioning this point because it allows to obtain the dose distribution due to a given number of seeds loaded in a plaque as a superposition of the dose distributions produced by a single seed in each one of the plaque positions.

\section{Plaque-seed modeling in Plaque Simulator system}

$T(r)$ can be obtained as the ratio of dose profile at central axis due to a single seed situated in a plaque to the same profile obtained for a single seed in a homogeneous water medium [6]. As no dependence on the plaque size has been found, the same factor could be used for all plaques. However Thomson and Rogers [12] suggested that $T(r)$ depends on the seed model. Table 5 shows values of $T(r)$ function introduced in the BPSS physical model.

Figure 6 compares dose profiles in central axis from MC simulation and from BPSS, normalized at $z=0.5 \mathrm{~cm}$. Profiles for one seed on the central position of the $12 \mathrm{~mm}$ plaque are logically coincident as the profile from MC was used to calculate $T(r)$ that is defined in the physics file. There is also a good agreement in the profiles found for the $12 \mathrm{~mm}$ plaque loaded with 8 seeds, with a maximum relative difference smaller than $2.5 \%$.

Off axis dose distribution obtained with the modified $T(r)$ function are shown in Fig. 7. A reasonable agreement is found in a relatively large area around the central axis where isodose curves differ at most by a few tenths of mm. Larger differences are found in the outer part of the dose curves, in the plaque penumbral region.

Table 4

I25.S06/I25.S16 Bebig seed 2D anisotropy function obtained in the MC simulations with PENELOPE.

\begin{tabular}{|c|c|c|c|c|c|c|c|c|c|c|c|c|}
\hline \multirow[b]{2}{*}{$\theta\left[^{\circ}\right]$} & \multicolumn{12}{|l|}{$r[\mathrm{~cm}]$} \\
\hline & 0.10 & 0.15 & 0.25 & 0.50 & 0.75 & 1.00 & 2.00 & 3.00 & 4.00 & 5.00 & 7.50 & 10.00 \\
\hline 0 & - & - & $0.31(1)$ & $0.41(2)$ & $0.47(2)$ & $0.494(5)$ & $0.571(6)$ & $0.602(7)$ & $0.630(8)$ & $0.647(7)$ & $0.68(1)$ & $0.69(1)$ \\
\hline 1 & - & - & $0.312(9)$ & $0.41(1)$ & $0.47(1)$ & $0.493(4)$ & 0.569 (5) & $0.604(6)$ & $0.632(7)$ & $0.648(6)$ & $0.680(9)$ & $0.70(1)$ \\
\hline 2 & - & - & $0.320(5)$ & $0.420(8)$ & $0.460(9)$ & $0.493(3)$ & $0.569(4)$ & $0.607(4)$ & $0.633(4)$ & $0.648(4)$ & $0.679(5)$ & $0.704(7)$ \\
\hline 3 & - & - & $0.332(5)$ & $0.422(7)$ & $0.467(7)$ & $0.496(2)$ & $0.570(3)$ & $0.607(3)$ & $0.631(3)$ & $0.647(3)$ & $0.676(4)$ & $0.703(6)$ \\
\hline 5 & - & - & $0.352(4)$ & $0.434(5)$ & $0.471(5)$ & $0.497(2)$ & $0.564(2)$ & $0.606(2)$ & $0.632(3)$ & $0.650(2)$ & $0.679(3)$ & $0.702(4)$ \\
\hline 7 & - & - & $0.381(3)$ & $0.442(4)$ & $0.475(5)$ & $0.504(1)$ & $0.576(2)$ & $0.614(2)$ & $0.642(2)$ & $0.659(2)$ & $0.693(3)$ & 0.709 (4) \\
\hline 10 & - & - & $0.442(3)$ & $0.476(4)$ & $0.514(4)$ & $0.545(1)$ & $0.614(1)$ & $0.650(2)$ & $0.672(2)$ & $0.686(2)$ & $0.714(2)$ & $0.730(3)$ \\
\hline 12 & - & - & $0.492(3)$ & $0.519(4)$ & $0.554(4)$ & $0.581(1)$ & $0.644(1)$ & $0.676(2)$ & $0.696(2)$ & 0.709 (2) & $0.733(2)$ & $0.748(3)$ \\
\hline 15 & - & - & 0.599 (3) & $0.586(3)$ & $0.614(4)$ & 0.637 (1) & $0.688(1)$ & 0.715 (1) & $0.732(2)$ & $0.743(1)$ & $0.761(2)$ & $0.774(3)$ \\
\hline 20 & - & $1.306(3)$ & $0.739(3)$ & $0.688(3)$ & $0.702(3)$ & $0.717(1)$ & $0.754(1)$ & $0.772(1)$ & $0.783(1)$ & $0.792(1)$ & $0.805(2)$ & $0.813(2)$ \\
\hline 25 & - & $1.080(3)$ & $0.826(3)$ & $0.762(3)$ & $0.772(3)$ & $0.7805(9)$ & $0.806(1)$ & $0.819(1)$ & $0.827(1)$ & $0.831(1)$ & $0.841(2)$ & $0.846(2)$ \\
\hline 30 & $1.737(5)$ & $0.990(3)$ & $0.881(3)$ & $0.820(3)$ & $0.826(3)$ & $0.8295(9)$ & $0.847(1)$ & $0.856(1)$ & $0.861(1)$ & $0.864(1)$ & $0.8708(8)$ & $0.875(2)$ \\
\hline 35 & $1.455(4)$ & $0.984(3)$ & $0.915(3)$ & $0.865(3)$ & $0.865(3)$ & $0.8679(9)$ & $0.8804(9)$ & $0.886(1)$ & $0.890(1)$ & $0.892(1)$ & $0.896(2)$ & $0.899(2)$ \\
\hline 40 & $1.269(4)$ & $0.981(3)$ & $0.939(3)$ & $0.901(3)$ & $0.896(3)$ & $0.8981(8)$ & $0.9062(9)$ & $0.911(1)$ & $0.913(1)$ & $0.914(1)$ & $0.917(2)$ & $0.919(2)$ \\
\hline 45 & $1.133(4)$ & $0.983(3)$ & $0.954(3)$ & $0.927(3)$ & $0.922(3)$ & $0.9228(8)$ & $0.9280(9)$ & $0.931(1)$ & $0.932(1)$ & $0.934(1)$ & $0.935(1)$ & $0.937(2)$ \\
\hline 50 & $1.031(3)$ & $0.986(3)$ & $0.965(3)$ & $0.947(3)$ & $0.944(3)$ & $0.9433(8)$ & $0.9463(8)$ & $0.9480(9)$ & $0.948(1)$ & 0.9489 (9) & 0.951 (1) & $0.952(2)$ \\
\hline 55 & $1.000(3)$ & $0.987(3)$ & $0.975(3)$ & $0.963(3)$ & $0.959(3)$ & $0.9587(8)$ & $0.9607(8)$ & 0.9619 (9) & $0.962(1)$ & $0.9613(9)$ & $0.964(1)$ & $0.964(2)$ \\
\hline 60 & $1.002(3)$ & $0.990(3)$ & $0.982(3)$ & $0.974(3)$ & $0.972(3)$ & $0.9713(8)$ & $0.9722(8)$ & $0.9730(9)$ & $0.972(1)$ & $0.9726(9)$ & $0.975(1)$ & $0.975(2)$ \\
\hline 65 & $1.002(3)$ & $0.992(3)$ & $0.987(3)$ & $0.986(3)$ & $0.980(3)$ & $0.9815(7)$ & $0.9814(8)$ & 0.9818 (9) & $0.982(1)$ & $0.9816(9)$ & $0.983(1)$ & $0.982(2)$ \\
\hline 70 & $1.000(3)$ & $0.995(3)$ & $0.993(3)$ & $0.990(3)$ & $0.988(3)$ & $0.9889(7)$ & $0.9887(8)$ & 0.9890 (9) & $0.989(1)$ & $0.9886(9)$ & $0.990(1)$ & $0.990(2)$ \\
\hline 73 & $1.003(3)$ & $0.998(3)$ & $0.994(3)$ & $0.993(2)$ & $0.993(2)$ & $0.9921(7)$ & $0.9920(8)$ & 0.9930 (9) & $0.993(1)$ & $0.9915(9)$ & $0.993(1)$ & $0.993(2)$ \\
\hline 75 & $1.000(3)$ & $0.995(3)$ & $0.996(3)$ & $0.994(2)$ & $0.992(2)$ & $0.9943(7)$ & $0.9948(8)$ & 0.9947 (9) & $0.994(1)$ & $0.9936(8)$ & 0.995 (1) & $0.994(2)$ \\
\hline 78 & $1.000(3)$ & $0.996(3)$ & 0.998 (3) & $0.996(3)$ & $0.996(2)$ & $0.9961(7)$ & $0.9967(8)$ & 0.9973 (9) & $0.997(1)$ & $0.9962(8)$ & $0.997(1)$ & $0.997(2)$ \\
\hline 80 & $1.000(3)$ & $0.997(3)$ & $0.997(3)$ & $1.000(3)$ & $0.996(2)$ & $0.9970(7)$ & $0.9976(8)$ & $0.9985(8)$ & $0.998(1)$ & $0.9972(8)$ & 0.999 (1) & $0.999(2)$ \\
\hline 82 & $1.001(3)$ & $0.999(3)$ & $0.998(3)$ & $1.000(3)$ & $1.000(2)$ & $0.9981(7)$ & $0.9990(8)$ & $0.9992(8)$ & $0.999(1)$ & $0.9982(8)$ & 0.999 (1) & $0.999(2)$ \\
\hline 84 & $1.001(3)$ & $0.998(3)$ & $1.001(3)$ & $1.000(3)$ & $0.997(2)$ & $0.9984(7)$ & $0.9993(8)$ & $0.9999(8)$ & $0.999(1)$ & $0.9992(8)$ & $1.000(1)$ & $1.001(2)$ \\
\hline 85 & $1.002(3)$ & 0.998 (3) & $1.001(3)$ & 0.999 (3) & $0.998(2)$ & $0.9985(7)$ & $0.9994(8)$ & $1.0004(8)$ & 0.999 (1) & $0.9997(8)$ & $1.000(1)$ & $1.001(2)$ \\
\hline 86 & $1.001(3)$ & $0.997(3)$ & $1.000(3)$ & 0.999 (3) & $0.999(2)$ & $0.9986(7)$ & $0.9998(8)$ & $1.0005(8)$ & $1.000(1)$ & $1.0001(8)$ & $1.001(1)$ & $1.000(2)$ \\
\hline 87 & $1.000(3)$ & $0.999(3)$ & $0.998(3)$ & 0.999 (3) & $0.999(2)$ & $0.9993(7)$ & $1.0003(8)$ & $1.0004(8)$ & $1.001(1)$ & $0.9997(8)$ & $1.001(1)$ & $1.000(2)$ \\
\hline 88 & $1.000(3)$ & $0.999(3)$ & 0.999 (3) & $1.000(3)$ & $1.000(2)$ & $1.0000(7)$ & $1.0001(8)$ & $1.0004(8)$ & $1.001(1)$ & $0.9995(8)$ & $1.002(1)$ & $1.000(2)$ \\
\hline 89 & $1.000(3)$ & $1.000(3)$ & $1.000(3)$ & $1.000(3)$ & $1.000(2)$ & $1.0001(7)$ & $0.9999(8)$ & $1.0002(8)$ & $1.000(1)$ & $0.9998(8)$ & $1.001(1)$ & $1.000(2)$ \\
\hline 90 & 1.0 & 1.0 & 1.0 & 1.0 & 1.0 & 1.0 & 1.0 & 1.0 & 1.0 & 1.0 & 1.0 & 1.0 \\
\hline
\end{tabular}



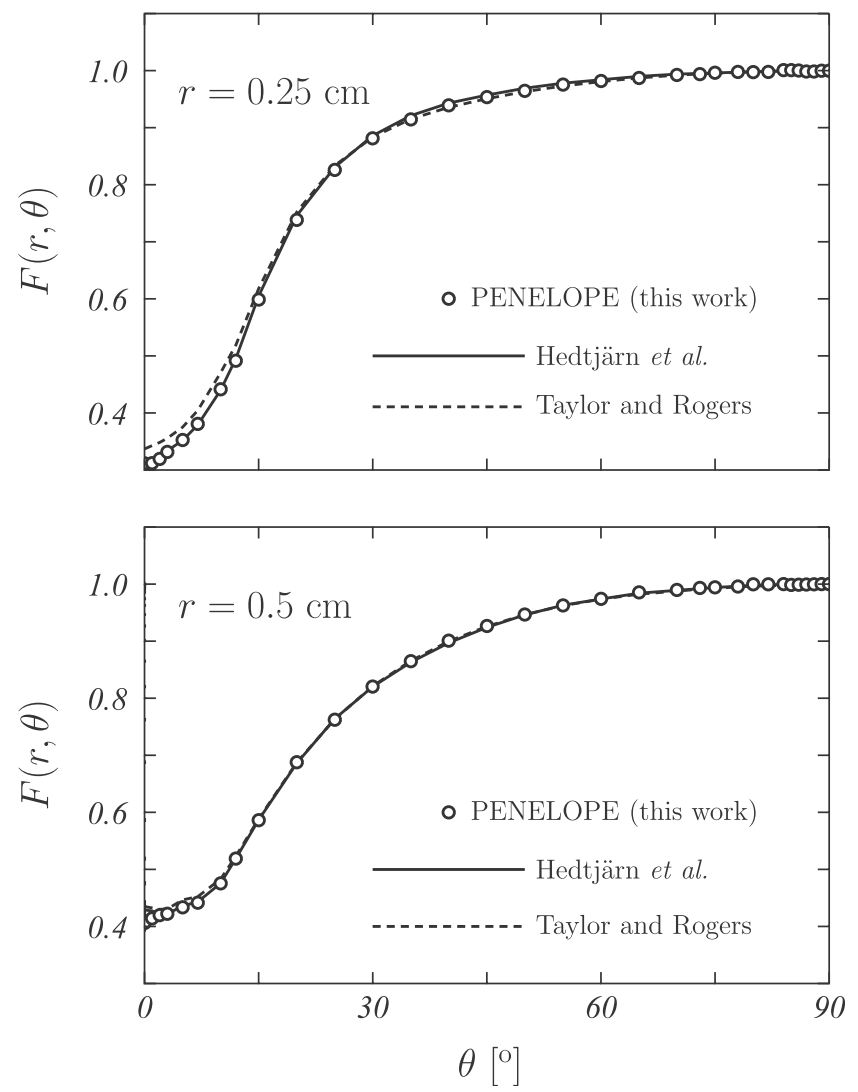

Figure 4. Anisotropy function found in the simulation for the I25.S06/I25.S16 Bebig seed (dots) compared to the results of Hedtjärn et al. [8] (solid curves) and Taylor and Rogers [20] (dashed curved) for $r=0.25$ (upper panel) and $0.5 \mathrm{~cm}$ (lower panel).

These differences are due to plaque collimation effects that are taken into account in Astrahan's algorithm using a simple geometrical penumbra model.

Recently, Rivard et al. [22] studied the dosimetry of COMS plaques loaded with the Oncura type 6711 seed model. For a $16 \mathrm{~mm}$ plaque, they calculated with the EGSnrc user-code

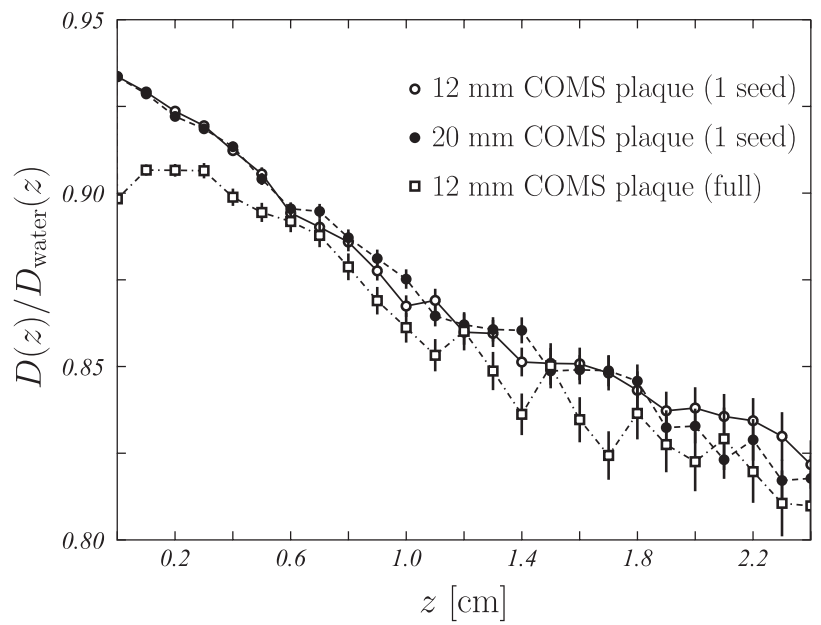

Figure 5. Ratio of dose distribution in the central axis for different configurations of COMS plaques to the corresponding dose distribution in a homogeneous water medium (without plaque). Open and solid circles correspond to a single seed loaded in the center of the $12 \mathrm{~mm}$ and $20 \mathrm{~mm}$ COMS plaques, respectively. Open squares correspond to the $12 \mathrm{~mm}$ plaque fully loaded.
Table 5

Values of $T(r)$ function introduced in the physical model of BPSS. They correspond to the ratio between plaque and water dose distributions in the central axis of a $12 \mathrm{~mm}$ plaque with a single seed in the central position. The value at $r=0$ has been fixed to 1.0 using the same criteria as in BPSS.

\begin{tabular}{ll}
\hline $\mathrm{r}[\mathrm{cm}]$ & $T(r)$ \\
\hline 0.0 & 1.0 \\
0.24 & 0.934 \\
0.34 & 0.929 \\
0.44 & 0.924 \\
0.54 & 0.919 \\
0.64 & 0.912 \\
0.74 & 0.906 \\
0.84 & 0.890 \\
1.04 & 0.886 \\
1.14 & 0.878 \\
1.24 & 0.867 \\
1.44 & 0.860 \\
1.64 & 0.851 \\
1.84 & 0.851 \\
2.04 & 0.843 \\
2.24 & 0.838 \\
2.44 & 0.835 \\
2.64 & 0.822 \\
\hline
\end{tabular}

BrachyDose the isodose contours for a configuration in which all seeds were fully modeled in water without interseed effects and another one in which the seeds and the plaque were fully modeled. Large differences (above 10\%) in all eye regions were found between both calculations. This points out the improvement that the modified $T(r)$ provides taking care to a large extent of the plaque effects.

Using BPSS, a treatment was calculated in which 85 Gy were delivered to the apex at $5 \mathrm{~mm}$ with the $12 \mathrm{~mm}$ plaque fully loaded with seeds of $S_{K}=6.6 \mathrm{U}$ at implant time. The resulting treatment time was $112 \mathrm{~h}$. This time was used to calculate the dose, at the same prescription point, using the MC dose rate obtained in the simulation. We found $84.64 \pm 0.37 \mathrm{~Gy}$, in agreement with the prescribed dose within statistical uncertainties.

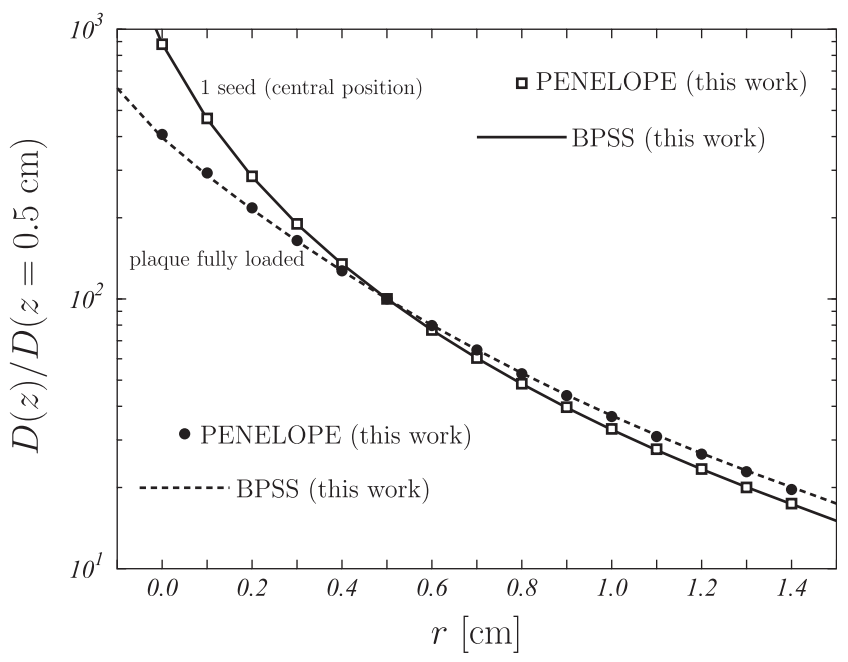

Figure 6. Dose distribution in the central axis for a $12 \mathrm{~mm}$ plaque calculated with $\mathrm{MC}$ simulation (symbols) and with BPSS (curves) for two different configurations corresponding to a seed situated in the central location (open squares) and to the plaque fully loaded (solid circles). 


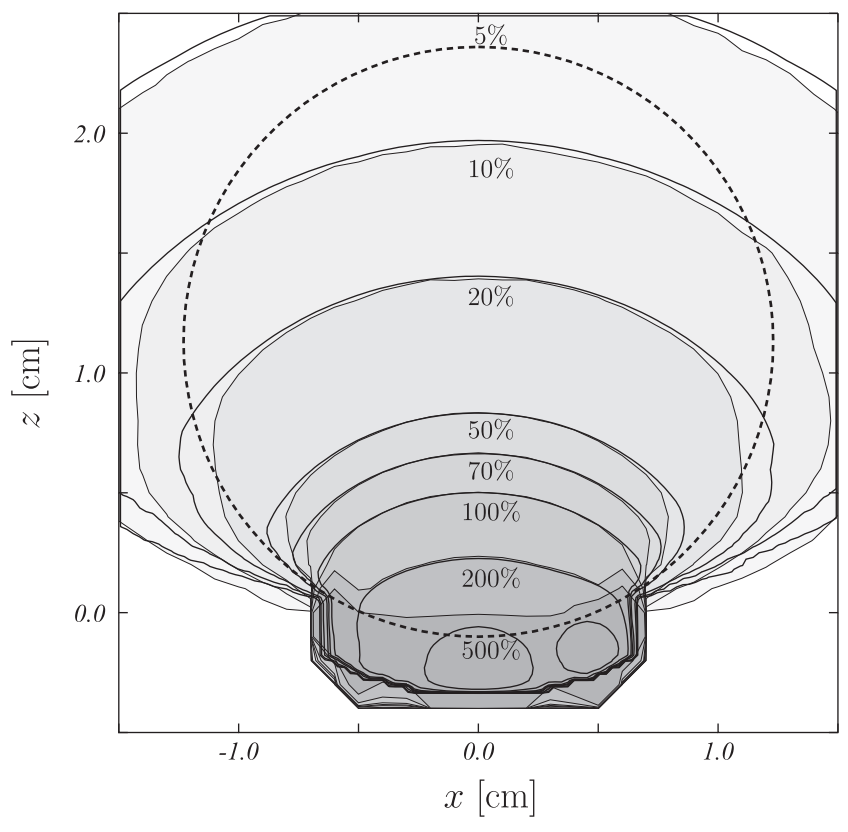

Figure 7. Comparison between isodose curves obtained in the MC simulation (thin solid curves) and those provided by BPSS (thick solid curves) in the $y=0$ plane for the $12 \mathrm{~mm}$ plaque fully loaded. Dose is normalized to the prescription dose point $(x=0$, $y=0, z=0.5 \mathrm{~cm})$. Dashed circumference shows the position of an eye with a diameter $24.6 \mathrm{~mm}$.

The same treatment was calculated with BPSS but using the default physics model, obtaining a value of $81.0 \mathrm{~Gy}$, that is $4.7 \%$ smaller. This difference is mainly related to the scatter and absorption correction function, $T(r)$, which in the default model is taken from the measurements performed with TLD by Chiu-Tsao et al. for a 6711 seed [3]. If one works with the apparent activity instead of air kerma strength, the conversion factor $\Gamma_{\delta}=S_{k} / A_{\mathrm{app}}$ provided by the manufacturer must be used and cannot be changed by other values even if they are more accurate [7,23].

\section{Conclusions}

COMS ophthalmic applicators loaded with I25.S16 seeds have been simulated using the MC code PENELOPE.

First, the I25.S16 seed, that shares the same geometry with the I25.S06 seed used in prostate brachytherapy, has been simulated and the dosimetric parameters defined in TG-43U1 protocol have been obtained and compared to results previously published by different authors. We have found a good agreement.

Dosimetry for various seed configurations in COMS plaques has been simulated to study the influence of the set plaque-seeds and the absorption among seeds on the dose distribution. Results are consistent with those previously published for other seed models.

Finally, the MC simulation results have been used to model the set plaque-seeds into the BPSS. This system allows the user to edit the default calculation parameters to create a customized model.
The doses calculated with BPSS and MC, for a $12 \mathrm{~mm}$ COMS plaque, agree within the uncertainty margin.

\section{Acknowledgments}

The work of AML has been supported in part by the Junta de Andalucía (FQM0220) and by the Spanish DGI (FPA2009-14091C02-02).

\section{References}

[1] Collaborative Ocular Melanoma Study Group. The COMS randomized trial of iodine 125 brachytherapy for choroidal melanoma. III. Initial mortality findings. COMS report No. 18. Arch Ophthalmol (Chicago) 2001;105:969-82.

[2] Nag S, Quivey JM, Earle JD, Followill DS, Fontanesi J, Finger PT. The American brachytherapy society recommendations for brachytherapy of uveal melanomas. Int J Radiat Oncol Biol Phys 2003;56:544-55.

[3] Chiu-Tsao S, Anderson LL, O'Brien K, Stabile L, Liu JC. Dosimetry for ${ }^{125}$ I seed (model 6711) in eye plaques. Med Phys 1993;20:383-9.

[4] De la Zerda A, Chiu-Tsao S, Lin J, Boulay LL, Kanna I, Kim JH. ${ }^{125} \mathrm{I}$ plaque dose distribution including penumbra characteristics. Med Phys 1996;23:407-18.

[5] Krintz AL, Hansons WF, Ibbott GS, Followill DS. A reanalysis of the collaborative ocular melanoma study medium tumor trial eye plaque dosimetry. Int J Radiat Oncol Biol Phys 2003;56:889-98.

[6] Astrahan MA. Improved treatment planning for COMS eye plaques. Int J Radiat Oncol Biol Phys 2005;61:1227-42.

[7] Rivard MJ, Coursey BM, DeWerd LA, Hanson WF, Saiful Huq M, Ibbott GS, et al Update of AAPM task group No. 43 report: a revised AAPM protocol for brachytherapy dose calculations. Med Phys 2004;31:633-74.

[8] Hedtjärn H, Carlsson GA, Williamson JF. Monte Carlo-aided dosimetry of the symmetric model I25.S06 ${ }^{125} \mathrm{I}$ interstitial brachytherapy seed. Med Phys 2000;27:1076-85.

[9] Sahoo S, Palani Selvam T. An EGSnrc investigation of the air-kerma strength dose rate constant, and radial dose function of ${ }^{125}$ I brachytherapy sources. Radiol Phys Technol 2009;2:198-204.

[10] Taylor RE, Rogers DW. An EGSnrc Monte Carlo-calculated database of TG-43 parameters. Med Phys 2008;35:4228-41.

[11] Salvat F, Fernández-Varea JM, Sempau J. PENELOPE - a code system for Monte Carlo simulation of electron and photon transport. Issy-les-Moulineaux:OECD Nuclear Energy Agency; 2008

[12] Thomson RM, Rogers DWO. Monte Carlo dosimetry for ${ }^{125} \mathrm{I}$ and ${ }^{103} \mathrm{Pd}$ eye plaque brachytherapy with various seed models. Med Phys 2010;37:368-76.

[13] Cullen DE, Hubbell JH, Kissel L. In: . EPDL97: the evaluated photon data library, '97 version, report UCRL-50400, vol. 6. Livermore: Lawrence Livermore National Laboratory; 1997. Rev. 5.

[14] Born M. Atomic physics. London: Blackie and Son; 1969.

[15] Hubbell JH, Veigele WJ, Briggs EA, Brown RT, Cromer DT, Mowerron KJ. Atomic form factors, incoherent scattering functions, and photon scattering cross sections. J Phys Chem Ref Data 1975;4:471-538.

[16] Ribberfors R. X-ray incoherent scattering total cross sections and energyabsorption cross sections by means of simple calculation routines. Phys Rev A 1983;27:3061-70.

[17] Taylor REP, Rogers DWO. The CRLP TG-43 parameter database. http://vali. physics.carleton.ca/clrp/seed_database/I125/IsoSeed_I25.S06/.

[18] Thomson RM, Taylor REP, Rogers DWO. Monte Carlo dosimetry for ${ }^{125} \mathrm{I}$ and ${ }^{103}$ Pd eye plaque brachytherapy. Med Phys 2008;35:5530-43.

[19] Rivard MJ, Granero D, Pérez-Calatayud J, Ballester F. Influence of photon energy spectra from brachytherapy sources on Monte Carlo simulations of kerma and dose rates in water and air. Med Phys 2010;37:869-76.

[20] Taylor REP, Rogers DWO. More accurate fitting of ${ }^{125} \mathrm{I}$ and ${ }^{103} \mathrm{Pd}$ radial dose functions. Med Phys 2008;35:4242-50.

[21] Patel NS, Chiu-Tsao S. Thermoluminescent dosimetry of the SymmetraTM ${ }^{125}$ model I25.S06 interstitial brachytherapy seed. Med Phys 2001;28:1761-9.

[22] Rivard MJ, Chiu-Tsao S, Finger PT, Meigooni AS, Melhus CS, Mourtada F, et al. Comparison of dose calculation methods for brachytherapy of intraocular tumors. Med Phys 2011;38:306-16.

[23] Williamson JF, Nath R. Clinical implementation of AAPM Task Group 32 recommendations on brachytherapy source strength specification. Med Phys 1991;18:439-48. 